\title{
Tumor Lysis Syndrome in Light Chain Multiple Myeloma Treated with Bortezomib Combination Therapy
}

\author{
Hasmik Minasyan, MD \\ Thomas Jefferson University, Hasmik.minasyan@jefferson.edu \\ Meaghan Henrici, MD \\ Thomas Jefferson University, meaghan.henrici@jefferson.edu
}

Follow this and additional works at: https://jdc.jefferson.edu/tmf
Part of the Medicine and Health Sciences Commons
Let uS know how access to this document benefits you

\section{Recommended Citation \\ Minasyan, MD, Hasmik and Henrici, MD, Meaghan (2015) "Tumor Lysis Syndrome in Light Chain Multiple Myeloma Treated with Bortezomib Combination Therapy," The Medicine Forum: Vol. 16 , Article 18. \\ DOI: https://doi.org/10.29046/TMF.016.1.017 \\ Available at: https://jdc.jefferson.edu/tmf/vol16/iss1/18}

This Article is brought to you for free and open access by the Jefferson Digital Commons. The Jefferson Digital Commons is a service of Thomas Jefferson University's Center for Teaching and Learning (CTL). The Commons is a showcase for Jefferson books and journals, peer-reviewed scholarly publications, unique historical collections from the University archives, and teaching tools. The Jefferson Digital Commons allows researchers and interested readers anywhere in the world to learn about and keep up to date with Jefferson scholarship. This article has been accepted for inclusion in The Medicine Forum by an authorized administrator of the Jefferson Digital Commons. For more information, please contact: JeffersonDigitalCommons@jefferson.edu. 


\title{
Tumor Lysis Syndrome in Light Chain Multiple Myeloma Treated with Bortezomib Combination Therapy
}

\author{
Hasmik Minasyan, MD* and Meaghan Henrici, MD* \\ *First authors
}

\section{INTRODUCTION}

Tumor lysis syndrome (TLS) is a potentially life threatening complication of cancer treatments that typically occurs in highly proliferative malignancies. It is rare in patients with multiple myeloma (MM) given the disease's indolent nature and is estimated to occur in less than $1 \%$ of cases. ${ }^{1}$ Increasing reports of TLS have been described in MM, particularly in treatment regimens containing bortezomib, the first available proteasome inhibitor. Here we describe a case of a newly diagnosed light chain multiple myeloma resulting in tumor lysis syndrome following the first dose of combination therapy with bortezomib, cyclophosphamide and dexamethasone.

\section{CASE REPORT}

A 68 year old man presented with 6-8 weeks of gradually worsening lower back pain and fatigue. In addition, he reported symptoms of severe constipation, nausea, night sweats, dysuria, right sided rib pain, and a $30 \mathrm{lb}$ weight loss. On examination, he had tenderness to palpation along the lower thoracic spine, para-spinal muscles and lower anterior ribs bilaterally. He was found to have deranged renal function with a serum creatinine of $2.75 \mathrm{mg} / \mathrm{dl}$, corrected serum calcium of $11.9 \mathrm{mg} / \mathrm{dl}$, macrocytic anemia with hemoglobin of $11.4 \mathrm{~g} / \mathrm{dl}$ (MCV 102) and urinalysis with trace protein but a urine protein to creatinine ratio of 9.6. The liver function tests were normal. An X-Ray of his thoracic and lumbar spine revealed a T12 compression fracture.

Subsequent labs revealed hypogammaglobulinemia and beta-2 microglobulin elevation of $19.53 \mathrm{mg} / \mathrm{L}$ (normal 1.31-2.6 mg/L). Serum protein electrophoresis had a monoclonal lambda light chain spike measuring 14,520 $\mathrm{mg} / \mathrm{L}$. 24 hour urine studies showed $11.5 \mathrm{~g}$ of protein and $12.1 \mathrm{~g}$ lambda light chains. A skeletal survey showed a questionable lucent lesion in the right 10th posterior rib. He underwent a bone marrow biopsy which showed 45.9\% plasma cells. FISH probe was negative for common cytogenetic abnormalities including $13 q$ deletion. The patient was diagnosed with lambda light chain multiple myeloma, International Staging System (ISS) stage III.
For treatment, the day 1 cycle included bortezomib $1.3 \mathrm{mg} / \mathrm{m}^{2}$, cyclophosphamide $300 \mathrm{mg}$, and dexamethasone $40 \mathrm{mg}$. Prior to starting chemotherapy, his serum creatinine had improved to $2.5 \mathrm{mg} / \mathrm{dL}$ with normal serum electrolytes. Calcium remained elevated at $11.2 \mathrm{mg} /$ $\mathrm{dL}$ despite hydration. His care was transferred to the Veteran's Hospital's Community Living Center for the duration of his chemotherapy.

24 hours after his first dose of chemotherapy, he re-presented to the emergency room with hypoxia, hypotension, tachycardia, and tachypnea. No fever was observed. Initial laboratory values were as follows: potassium $5.5 \mathrm{mmol} / \mathrm{L}$, serum uric acid $22.7 \mathrm{mg} / \mathrm{dL}$, serum phosphorous $8.2 \mathrm{mg} / \mathrm{dL}$, bicarbonate $20 \mathrm{mmol} / \mathrm{L}$ with anion gap of 17 , serum calcium $8.3 \mathrm{mg} / \mathrm{dL}$, lactate $1.7 \mathrm{mmol} / \mathrm{L}, \mathrm{LDH} 1147 \mathrm{IU} / \mathrm{L}$, and serum creatinine 3.41 $\mathrm{mg} / \mathrm{dL}$. He met clinical and laboratory criteria for tumor lysis syndrome (TLS) based on the Cairo-Bishop definition (Table 1). For TLS treatment the patient was started on IV fluid hydration with bicarbonate supplementation, allopurinol $200 \mathrm{mg}$ daily and given a dose of raspuricase $0.15 \mathrm{mg} / \mathrm{kg}$. The patient was admitted to the ICU and closely monitored.

TABLE 1: CAIRO-BISHOP DEFINITION OF TUMOR LYSIS SYNDROME (TLS) IN ADULTS

Laboratory TLS: $\geq 2$ of the following serum abnormalities, developing within 3 days before or 7 days after initiation of chemotherapy

-Uric acid $\geq 8 \mathrm{mg} / \mathrm{dL}$ or $25 \%$ increase from baseline

-Potassium $\geq 6 \mathrm{meq} / \mathrm{L}$ or $25 \%$ increase from baseline

-Phosphorous $\geq 4.5 \mathrm{mmol} / \mathrm{dL}$ or $25 \%$ increase from baseline

-Calcium $\leq 7 \mathrm{mg} / \mathrm{dL}$ or $25 \%$ decrease from baseline

Clinical TLS: Laboratory TLS AND $\geq 1$ of the following criteria

-Creatinine $>1.5 x$ upper limit of normal

-Cardiac arrhythmia or sudden death

-Seizure 


\section{The Medicine Forum, Vol. 16 [2015], Art. 18}

\section{DIFFERENTIAL DIAGNOSIS}

Differential diagnosis of new onset lower back pain in this elderly man included multiple myeloma, metastatic prostate cancer, lumbar strain, hyperparathyroidism and severe constipation secondary to an underlying malignancy of the colon.

\section{OUTCOME AND FOLLOW UP}

Over the course of several days, the patient's TLS labs normalized and he was discharged back to the Veteran's Community Living Center for rehabilitation. Per oncology's recommendation, he was treated again with bortezomib, cyclophosphamide and dexamethasone. He was re-admitted to the hospital approximately 2 weeks later with altered mental status and dehydration. Tumor lysis labs at this time were normal. During this hospitalization, the patient and his family made the decision to withhold further treatment and enter home hospice.

\section{DISCUSSION}

Tumor lysis syndrome (TLS) is a complication carrying a high morbidity and mortality most commonly occurring secondary to cell lysis caused by chemotherapy with the subsequent release of intracellular potassium, phosphate, and nucleic acids into the bloodstream. Characteristic laboratory findings include hyperkalemia, hyperphosphatemia, hyperuricemia, hypocalcemia and elevated LDH. Clinically, patients can present with nondescript symptoms such as nausea and vomiting, or life threatening cardiac arrhythmias, renal failure, seizures,or sudden death. TLS is most frequently associated with highly proliferative neoplasms such as non-Hodgkins lymphoma and acute lymphocytic leukemia, while plasma cell dyscrasias such as multiple myeloma are classified as typically having a $<1 \%$ risk. ${ }^{1}$

Multiple myeloma is characterized by a low proliferative index with less than $1 \%$ plasma cells engaging in cellular proliferation. ${ }^{2}$ As a result, TLS is a rare complication of chemotherapy in the treatment of multiple myeloma. It is believed to have a higher likelihood of occurrence in patients with a high tumor burden, especially if treated with bortezomib in combination with other agents. The definition of high tumor cell burden is not clear in the literature, but it is believed to be correlated with increased serum lactate dehydrogenase and Beta-2 microglobulin levels ( $\geq 5.5 \mathrm{mg} / \mathrm{dL}$ ) as well as diffuse bone marrow disease, multiple lytic lesions, hypercalcemia (>12 mg/dL) and positive $\mathrm{C}$ reactive protein. ${ }^{8}$ TLS has also been cited to occur in monotherapy treatment with bortezomib, steroids, or thalidomide. ${ }^{2}$ Additional risk factors associated with the development of TLS in multiple myeloma include high proliferative activity, immature plasma cell morphology, and poor cytogenetics. 2.3 .4 Case reports by Hung Chang et al associate higher rates of TLS in light chain myeloma with pre-existing renal insufficiency prior to chemotherapy, a presentation similar to our patient. Nonetheless, not enough data exists to have clear understanding of the risk factors and whether certain types of multiple myeloma carry a higher risk.

Since the introduction of bortezomib, there have been several other case reports of patients with multiple myeloma developing tumor lysis syndrome with a reported incidence of $1.4 \%{ }^{3}$ As a reversible inhibitor of the 265 proteasome, bortezomib's impact on the transcriptional factor NF-kB is thought to be the mechanism of rapidly induced cancer cell apoptosis. ${ }^{3}$ In a case series, Sezer et al. reviewed 496 cases of multiple myeloma treated with bortezomib in three phase II multi-center trials and found that the criteria for tumor lysis syndrome was fulfilled in 7 cases (1.8\%). TLS caused renal failure leading to dialysis occurred in three cases, with one patient subsequently dying from renal failure. When a timeline was explicitly described, the earliest TLS case occurred on day 5 following bortezomib administration, making our patient's presentation one day after treatment even more atypical. Additionally, in two of the cases, TLS did not occur on initial exposure to bortezomib, but rather during retreatment with bortezomib in combination with dexamethasone.

Since the majority of reported cases of TLS typically occur very early in the course of treatment with bortezomib, patients are at the highest risk during the first cycle of therapy. Therefore, it is important to closely monitor patients receiving bortezomib, particularly those with higher tumor burden. Concomitant use of thalidomide or dexamethasone, such as in our patient, tends to increase the likelihood of tumor lysis syndrome. ${ }^{5}$ Chim in his article suggests TLS prophylaxis with hydration and alkalinization in patients with a high tumor burden who are being started on a treatment with bortezomib. 


\section{CONCLUSION}

Although rare, tumor lysis syndrome (TLS) is a potentially severe complication in the treatment of multiple myeloma. After initiation of treatment, patients should be closely monitored, particularly those with a high tumor burden receiving bortezomib. There is a need for further studies and case reports to identify incidence and predicting factors that may lead to TLS in patients treated for multiple myeloma.

\section{REFERENCES}

1. Cairo MS, Coiffier B, Reiter A. Recommendations for the evaluation of risk and prophylaxis of tumour lysis syndrome (TLS) in adults and children with malignant diseases: an expert TLS panel consensus. Br J Haematol 2010; 149:578.

2. Chang H, Lee SY, Tang TC. Tumor Lysis Syndrome in Patients with Light Chain Multiple Myeloma: Report of Two Cases. Chang Gung Med J 2011;34(6 Suppl):70-5)

3. Chim C.S. Rapid Complete remission in multiple myeloma with bortezomib/thalidomide/dexamethasone combination therapy following development of tumor lysis syndrome. Cancer Chemother Pharmacol (2008) 62:181-182.

4. Dhanraj KM, Biswajit D. Tumor lysis syndrome in multiple myeloma treated with bortezomib. J Pharmacol Pharmacother 2014;5:161-2.

5. Kenealy MK, Prince HM, Honemann D. Tumor Lysis Syndrome Early After Treatment with Bortezamib for Multiple Myeoloma. Pharmacotherapy 2006; 26(8):1205-1206.

6. Sezer $\mathrm{O}$, Vesole DH, Singhal S,...Mehta J. Bortezomib-Induced Tumor Lysis Syndrome in Multiple Myeloma. Clinical Lymphoma \& Myeloma 2006; 7(3):233-235

7. Terpos E, Politou M, Rahemtulla A. Tumour lysis syndrome in multiple myeloma after bortezomib (VELCADE) administration. J Cancer Res Clin Oncol 2004; 130:623-625.

8. National Comprehensive Cancer Network. Clinical Practice guidelines in Oncology: Multiple Myeloma. V.1.2011.

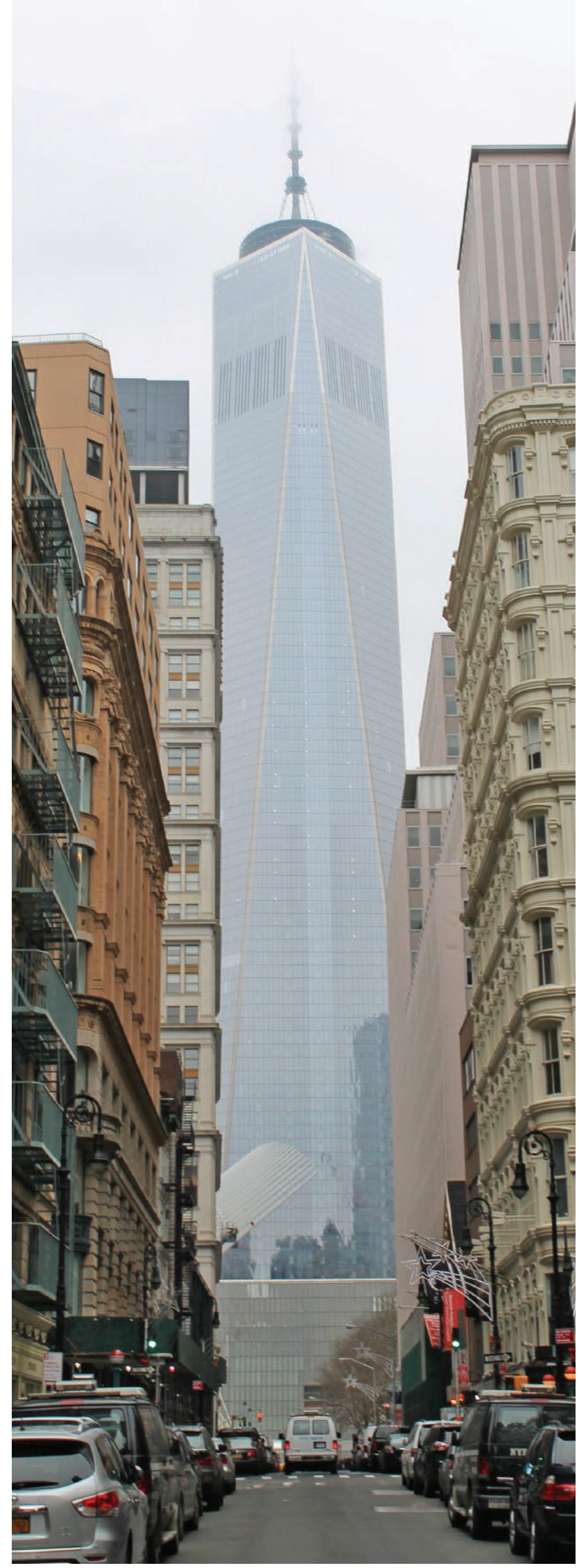

